\title{
THE BORDISM RING OF MANIFOLDS WITH INVOLUTION
}

\author{
J. C. ALEXANDER
}

\begin{abstract}
A complete set of representatives is found for the bordism ring of manifolds with involution. The multiplicative structure is expressed in terms of these representatives, and in particular, a set of ring generators can be isolated. Also the constructions involved are used to give an insight into a map of J. M. Boardman.
\end{abstract}

1. Introduction. In [3], P. E. Conner and E. E. Floyd defined the notion of cobordism of differentiable manifolds with involution, and they investigated the structure of the resulting bordism groups. If $M$ is a closed differentiable manifold and $T: M \rightarrow M$ is an involution, $(M, T)$ bounds if there exists a manifold $N$ such that $M=\partial N$ and an involution $S: N \rightarrow N$ such that $T=S \mid M$. We let $\mathscr{I}=\left\{\mathscr{I}_{n}\right\}$ be the resulting bordism groups graded by dimension.

If $\mathscr{N}=\left\{\mathscr{N}_{n}\right\}$ is the unoriented bordism ring, we consider $\mathscr{N}$ a subgroup of $\mathscr{I}$ by $[M] \mapsto[M, I]$, where $I$ is the trivial involution. Similarly, we have an augmentation $\varepsilon: \mathscr{I} \rightarrow \mathscr{N}$ defined by $[M, T] \mapsto[M]$. The ring $\mathscr{N}$ operates on $\mathscr{I}$ by $[N] \cdot[M, T]=[N \times M, I \times T] . \mathscr{I}$ is a free $\mathscr{N}$-module. Indeed, $\mathscr{I}$ is a commutative ring, if we let

$$
\left[N, T_{1}\right]\left[M_{2}, T_{2}\right]=\left[M_{1} \times M_{2}, T_{1} \times T_{2}\right] .
$$

Conner and Floyd completely determined the additive structure of $\mathscr{I}$ [3, Theorem 28.1] which is as follows: there is a short exact sequence of $\mathscr{N}$ modules

$$
0 \longrightarrow \mathscr{I} \stackrel{\nu}{\longrightarrow} \mathscr{M} \stackrel{J}{\longrightarrow} \mathscr{N}_{*}\left(P^{\infty}\right) \longrightarrow 0,
$$

where $\mathscr{M}_{n}=\sum_{j=0}^{n} \mathscr{N}_{j}(B O(n-j))$. The notation is standard: $\mathscr{N}_{*}$ is the unoriented bordism (homology) theory, $B O(r)$ is the classifying space for real $r$-plane bundles, and $P^{\infty}=B O(1)$ is infinite-dimensional real projective space. The map $v$ is defined as follows: given $\left(M^{n}, T\right)$, let $F_{i}$ be the $i$-dimensional fixed-point submanifold of $M$, and let $\nu_{i}: F_{i} \rightarrow B O(n-i)$ be

Received by the editors March 26, 1971.

AMS 1970 subject classifications. Primary 57D85; Secondary 57E99.

Key words and phrases. Manifolds with involution, equivariant bordism, bordism $J$-homomorphism.

(c) American Mathematical Society 1972 
the normal bundle of $F_{i}$ in $M$. Then $\left[v_{i}\right] \in \mathscr{N}_{i}(B O(n-i))$, and $\nu[M, T]$ is the sum of these classes. Note that $\nu$ is a map of degree zero. $J$ is defined as follows: given an $r$-plane bundle $\eta$ over a manifold $M^{n}$, let $R P(\eta)$ be the total space of the associated projective-space bundle (an $(n+r-1)$ dimensional manifold), and let $\hat{\eta}$ over $R P(\eta)$ be the canonical line bundle. $J(\eta)$ is the class of $\hat{\eta}: R P(\eta) \rightarrow P^{\infty}$. The map $J$ is of degree -1 .

In this note, we exhibit a complete set of representatives for $\mathscr{I}$, and we determine its ring structure. Let $P^{n}=\left\{\left[x_{0}, \cdots, x_{n}\right]\right\}$ be real $n$-dimensional projective space, and let $T_{0}: P^{n} \rightarrow P^{n}$ be defined by $T_{0}\left[x_{0}, x_{1}, \cdots, x_{n}\right]=$ $\left[-x_{0}, x_{1}, \cdots, x_{n}\right]$. Denote the pair $\left(P_{n}, T_{0}\right)$ by $P_{0}^{n}$. We need a map $\Gamma: \mathscr{I} \rightarrow \mathscr{I}$ of degree +1 , defined as follows: let $S^{1}$ denote the circle $|z|=1$ in the complex plane. Given $(M, T)$, let $M^{\prime}=M \times S^{1} / \sim$, where $(m, z) \sim$ $(T m,-z)$. Define $T^{\prime}: M^{\prime} \rightarrow M^{\prime}$ by $T^{\prime}(m, z)=(T m, \bar{z})$. Then $\Gamma[M, T]=$ $\left[M^{\prime}, T^{\prime}\right]$ (compare $\left.[4, \S 4]\right)$.

\subsection{THEOREM. The set}

$$
\{1\} \cup\left\{\Gamma^{n}\left[P_{0}^{n_{1}} \times \cdots \times P_{0}^{n_{r}}\right] \mid n \geqq 0, r \geqq 1, n_{i} \geqq 2\right\}
$$

is a free $\mathscr{N}$-basis for $\mathscr{I}$.

The product of $\mathscr{I}$ is thus determined when we know how it interacts with $\Gamma$.

1.3. Theorem. If $a, b \in \mathscr{I}$, then

$$
\Gamma(a b)=\Gamma(a) \cdot b+\varepsilon(a) \cdot \Gamma(b)=a \cdot \Gamma(b)+\Gamma(a) \cdot \varepsilon(b) .
$$

Let $\theta$ be transcendental over $\mathscr{N}$; we consider the ring of formal power series $\mathscr{N}[[\theta]]$. Define $\tilde{J}: \mathscr{I} \rightarrow \mathscr{N}[[\theta]]$ by $\tilde{J}\left[M^{n}, T\right]=\sum_{r=0}^{\infty} \varepsilon \Gamma^{r}\left[M^{n}, T\right] \cdot \theta^{n+r}$ (the coefficient of $\theta^{s}$ is in $\mathscr{N}_{s}$ ).

\subsection{Corollary. $\tilde{J}$ is a ring homomorphism.}

J. M. Boardman (unpublished; see [1], [2] for a related map) has defined a ring homomorphism

$$
J^{\prime}: \mathscr{F}=\sum_{i=0}^{\infty} \mathscr{N}_{i}(B O) \rightarrow \mathscr{N}[[\theta]],
$$

where $\mathscr{F}$ has the ring structure given by the external product of bundles. Let $\iota_{*}: \mathscr{N}_{i}(B O(r)) \rightarrow \mathscr{N}_{i}(B O)$ be induced by inclusion. Then $\iota_{*} v$ is a ring homomorphism. We connect our results with Boardman's by the following result.

\subsection{THEOREM. $\tilde{J}=J^{\prime} \iota_{*} v$.}

Although most of the results in this note are independent of Boardman's, they follow from an attempt to understand his work, and I would like to 
thank him for several discussions about his work. Also, R. E. Stong has shown me some of his unpublished work wherein he obtains essentially the same result as our Theorem 1.2.

2. The map $\Gamma . \quad \Gamma: \mathscr{I}_{n} \rightarrow \mathscr{I}_{n+1}$ is defined as follows: let $S^{1}$ denote the circle $|z|=1$ in the complex plane. Given a pair $(M, T)$, let $M^{\prime}=M \times S^{1} / \sim$, where $(m, z) \sim(T m,-z)$. The involution $T^{\prime}$ is defined on $M^{\prime}$ by $T^{\prime}(m, z)=$ $(T m, \bar{z})$. Then $\Gamma[M, T]=\left[M^{\prime}, T^{\prime}\right]$. These manifolds $\left(M^{\prime}, T^{\prime}\right)$ (and $M^{(r)}$, $\left.T^{(r)}\right)$ are called $V(n, k)$ in [4]. Alternatively, if $I=[0,1]$, let $M^{\prime}=M \times I / \sim$, where $(m, 0) \sim(T m, 1)$. In this notation, $T^{\prime}$ is defined by $T^{\prime}(m, t)=(T m$, $1-t)$.

It is easily verified that (1) $\Gamma$ is well defined, (2) $\Gamma$ is additive, (3) $\Gamma$ is an $\mathscr{N}$-map, (4) $\Gamma(\mathscr{N})=0$.

If $F$ is the fixed-point set of $(M, T)$ and $\nu$ is the normal bundle of $F \subset M$, the fixed point set of $\left(M^{\prime}, T^{\prime}\right)$ is $F \cup M$ (here $F \equiv F \times \frac{1}{2} \subset F \times I ; M \equiv$ $M \times 0 \subset M \times I)$ with normal bundles $\nu \oplus o^{1} \cup o^{1}$, where $o^{r}$ is the trivial $r$ plane bundle. The natural inclusion $\iota: B O(r) \rightarrow B O(r+1)$ induces $\iota_{*}$ : $\mathscr{N}(B O(r)) \rightarrow \mathscr{N}(B O(r+1))$, and the map $M \rightarrow$ (base point) induces $\tau$ : $\mathscr{N}_{n} \rightarrow \mathscr{N}_{n}(B O(1))$.

\subsection{Proposition. $v \Gamma=\iota_{*} \nu+\tau \varepsilon$.}

Since $\iota_{*}$ is an injection, the next result is immediate.

2.2. Corollary. If $[M, T] \notin \mathscr{N} \subset \mathscr{I}$, then $\Gamma[M, T] \notin \mathscr{N}$. In particular, $\Gamma[M, T]=0$ if and only if $[M, T] \in \mathscr{N}$.

1.3'. Theorem. For $\left[M_{i}, T_{i}\right] \in \mathscr{I}(i=1,2)$, $\Gamma\left(\left[M_{1}, T_{1}\right] \cdot\left[M_{2}, T_{2}\right]\right)=\left[M_{1}, T_{1}\right] \cdot \Gamma\left[M_{2}, T_{2}\right]+\Gamma\left[M_{1}, T_{1}\right] \cdot \varepsilon\left[M_{2}, T_{2}\right]$.

Proof. By (1.1), we need to compare bordism classes of fixed-point bundles. Let $\left(M_{i}, T_{i}\right)$ have fixed-point sets $F_{i}$ with normal bundles $v_{i}$. The fixed-point bundle of $\left(\left(M_{1}, T_{1}\right) \times\left(M_{2}, T_{2}\right)\right)^{\prime}$ is

$$
\left(\left(\nu_{1} \times \nu_{2}\right) \oplus o^{1}\right) \cup o^{1} \text { over }\left(F_{1} \times F_{2}\right) \cup\left(M_{1} \times M_{2}\right) .
$$

The fixed-point bundle of $\left(M_{1}, T_{1}\right) \times\left(M_{2}^{\prime}, T_{2}^{\prime}\right)$ is

$$
\left(\left(v_{1} \times v_{2}\right) \oplus o^{1}\right) \cup\left(v_{1} \times o^{1}\right) \text { over }\left(F_{1} \times F_{2}\right) \cup\left(F_{1} \times M_{2}\right) .
$$

The fixed-point bundle of $\left(M_{1}^{\prime}, T_{1}^{\prime}\right) \times\left(M_{2}\right.$, trivial $)$ is

$$
\left(v_{1} \oplus o^{1}\right) \cup o^{1} \text { over }\left(F_{1} \times M_{2}\right) \cup\left(M_{1} \times M_{2}\right) .
$$

The theorem now follows if we take bordism classes.

$1.4^{\prime}$. CoRollary. $\tilde{J}: \mathscr{I} \rightarrow \mathscr{N}[[\theta]]$ is a ring homomorphism. 
This is an easy consequence of a fomal manipulation with coefficients of power series.

3. $Z_{2} \otimes_{\mathscr{N}} \mathscr{I}$. Let $Z_{2}$ be the trivial $\mathscr{N}$-module concentrated in grading 0 . For an $\mathscr{N}$-module $\mathscr{R}$, let $\mathscr{R}=Z_{2} \otimes_{\mathscr{N}} \mathscr{R}$ which is a $Z_{2}$-module. If $f$ : $\mathscr{R}_{1} \rightarrow \mathscr{R}_{2}$ is a map of $\mathscr{N}$ modules, let $\hat{f} \widehat{\mathscr{R}}_{1} \rightarrow \widehat{\mathscr{R}}_{2}$ be the induced $Z_{2}$ map.

Since all terms in (1.1) are free $\mathscr{N}$-modules, we have an exact sequence

$$
0 \longrightarrow \hat{\mathscr{I}} \stackrel{\hat{v}}{\longrightarrow} \hat{\mathscr{M}} \stackrel{\mathcal{J}}{\longrightarrow}\left(\mathscr{N}_{*}\left(P^{\infty}\right)\right)^{\wedge} \longrightarrow 0 .
$$

Since $\left(\mathscr{N}_{*}(X)\right)^{\wedge}=H_{*}(X)\left(=H_{*}\left(X ; Z_{2}\right)\right)$ for each space $X$, we get the exact sequence

$$
0 \longrightarrow \hat{\mathscr{I}}_{n} \stackrel{\hat{v}}{\longrightarrow} \sum_{j=0}^{n} H_{j}(B O(n-j)) \stackrel{\hat{J}}{\longrightarrow} H_{n-1}\left(P^{\infty}\right) \longrightarrow 0 .
$$

Let $\iota_{*}: H_{*}(B O(r)) \rightarrow H_{*}(B O(r+1))$ be induced by the natural inclusion. The next two results are immediate consequences of Proposition (2.1).

3.3. Proposition. $\hat{\nu} \hat{\Gamma}=\iota_{*} \hat{v}$ in positive gradings.

3.4. COROllary. $\hat{\Gamma}$ is injective in positive gradings.

Let $[M, T]$ denote the class of $(M, T)$ in $\mathscr{I}$.

3.5. Corollary. $[M, T]$ eimage $\hat{\Gamma}$ implies $\hat{v}[M, T]$ eimage $\iota_{*}$.

$\hat{\nu}: \hat{\mathscr{I}} \rightarrow \hat{M}$ is the following map: Let $F_{i}$ be the $i$-dimensional fixedpoint set of $\left(M^{n}, T\right)$. Let $\left\{F_{i}\right\} \in H_{i}\left(F_{i}\right)$ be the fundamental class, and let $v_{i}: F_{i} \rightarrow B O(n-i)$ be the normal bundle of $F_{i} \subset M^{n}$. Then $\hat{v}[M, T]=$ $\sum\left(v_{i}\right)_{*}\left\{F_{i}\right\}$.

Since $\mathscr{I}$ is a two-sided $\mathscr{N}$ module, $\mathscr{I}$ inherits a ring structure. Further, the sum of bundles induces maps

$$
H_{j}(B O(n-j)) \otimes H_{k}(B O(m-k)) \rightarrow H_{j+k}(B O(m+n-j-k)) ;
$$

i.e., $\hat{\mathscr{M}}_{n} \otimes \hat{\mathscr{M}}_{m} \rightarrow \hat{\mathscr{M}}_{m+n}$. Thus $\hat{\mathscr{M}}$ is a graded ring. The map $\hat{v}$ is a ring homomorphism. Let $y_{j}$ be the nonzero element in $H_{j}\left(P^{\infty}\right)$, let

$$
\lambda: H_{j}\left(P^{\infty}\right)=H_{j}(B O(1)) \subset \hat{\mathscr{M}}_{j+1}
$$

be the inclusion, and let $x_{j}=\lambda y_{j-1}$. Since the $y_{j}(j \geqq 1)$ are algebraically independent in $H_{*}(B O)$, the $x_{j}(j \geqq 1)$ are algebraically independent in $\hat{M}$.

\subsection{Proposition. $\hat{\mathscr{M}}=Z_{2}\left[x_{1}, x_{2}, \cdots\right]$.}

Proof. The remarks above establish that $Z_{2}\left[x_{1}, x_{2}, \cdots\right]$ is a subring of $\hat{\mathscr{M}}$. The result is established once we count the dimensions (over $Z_{2}$ ) of 
the two sides. Let $\pi(r)$ be the number of partitions of the integer $r$. We claim

$$
\operatorname{dim} \mathscr{M}_{r}=\pi(r)
$$

this will finish the proof.

Let $\pi(r ; k)$ denote the number of partitions of $r$ with largest element precisely $k$. Formula (3.7) follows immediately from the following three facts (the verifications of which we leave to the reader):

(1) $\pi(r)=\sum_{k=1}^{r} \pi(r ; k)$,

(2) $\pi(r ; k)=\pi_{k}(r-k)$, where $\pi_{k}(n)$ is the number of partitions of $n$ with elements at most $k$,

(3) $\operatorname{dim}_{\mathbf{z}_{2}} H_{j}(B O(n-j))=\pi_{n-j}(j)$.

1.2'. THEOREM. $\{1\} \cup\left\{\hat{\Gamma}^{n}\left(P_{0}^{n_{1}} \times \cdots \times P_{0}^{n_{r}}\right) \mid n \geqq 0, r \geqq 1, n_{i} \geqq 2\right\}$ form $a$ $Z_{2}$-basis of $\hat{\mathscr{I}}$.

Proof. First we establish that these elements are linearly independent. It is sufficient, by Corollary (3.4), to establish that $\left\{P_{0}^{2}, P_{0}^{3}, \cdots\right\}$ are algebraically independent. The fixed point set of $P_{0}^{n}$ is the set $P^{n-1} \cup P^{0}$ with normal bundle $\xi_{n-1} \cup o^{n}$ over $P^{n-1} \cup P^{0}$. Here $\xi_{n-1}$ is the canonical bundle. Thus $\tilde{\nu} P_{0}^{n}=x_{n}+x_{1}^{n}$. By Proposition (3.6), $\left\{P_{0}^{2}, P_{0}^{3}, \cdots\right\}$ are algebraically independent.

Now we count the number of elements and compare it with the number required by (3.2) and (3.7). We use generating functions in $Z[[x]]$. Let $d_{n}(m)$ denote the number of elements

$$
\left\{\hat{\Gamma}^{m}\left[P_{0}^{n_{1}} \times \cdots \times P_{0}^{n_{r}}\right] \mid r>0, n_{i} \geqq 2\right\}
$$

in $\hat{\mathscr{I}}$, and let $d_{n}=\sum_{m=0}^{\infty} d_{n}(m)$.

Clearly,

Thus

$$
1+\sum_{n=1}^{\infty} d_{n}(0) x^{n}=\prod_{r \geq 2} \frac{1}{1-x^{r}}
$$

and

$$
\sum_{n=0}^{\infty} d_{n}(m) x^{n}=x^{m} \prod_{r \geq 2} \frac{1}{1-x^{r}}-x^{m}
$$

$$
\begin{aligned}
1+\sum_{n=1}^{\infty} d_{n} x^{n} & =\left(1+x+x^{2}+\cdots\right) \prod_{r \geqq 2} \frac{1}{1-x^{r}}-\left(x+x^{2}+x^{3}+\cdots\right) \\
& =\prod_{r \geqq 1} \frac{1}{1-x^{r}}-\frac{x}{1-x}=1+\sum_{n=1}^{\infty}(\pi(n)-1) x^{n} .
\end{aligned}
$$

The theorem is proved.

Using this result, we can improve some earlier results. 
Note that since $\hat{\Gamma}(a \cdot b)=a \cdot \hat{\Gamma}(b)$ (from $(1.3)), \hat{\Gamma} \hat{I}$ is an ideal in $\hat{I}$. The most elemental collection of elements seems to be $\hat{\mathscr{I}} / \hat{\Gamma} \hat{\mathscr{I}}$.

3.8. Corollary. $\hat{\mathscr{I}} / \hat{\Gamma} \hat{\mathscr{I}}=Z_{2}\left[P_{0}^{2}, P_{0}^{3}, \cdots\right]$.

3.9. Corollary. $\quad[M, T] \in \hat{\Gamma} \hat{I}$ if and only if $\nu[M, T]$ eimage $\iota_{*}$.

4. Connection with Boardman's results. First we give a definition of Boardman's map $J^{\prime}$. The diagram

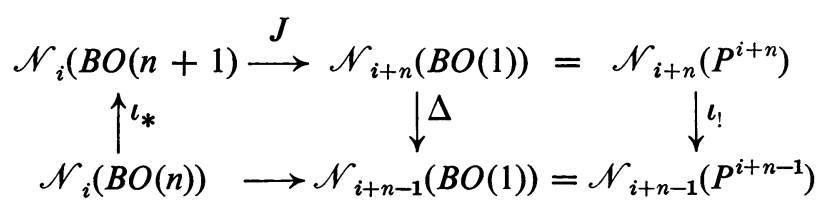

is commutative [3, Theorem 26.4]. Here $\Delta$ is defined as in [3], and $\iota_{1}$ is the bordism dual to the map $\iota^{*}$ induced in cobordism by inclusion. If for $N \geqq r, \kappa_{r, N}: P^{r} \rightarrow P^{N}$ is the natural inclusion, then

$$
\begin{aligned}
\iota_{!}\left[\kappa_{r, N}\right] & =0 & & (r=0), \\
& =\left[\kappa_{r-1, N-1}\right] & & (r>0) .
\end{aligned}
$$

The $\left\{\left[\kappa_{r, N}\right] \mid 0 \leqq r \leqq N\right\}$ constitute a free $\mathscr{N}$-basis for $\mathscr{N}\left(P^{N}\right)$. Also, if $\theta_{N}=\left[\kappa_{N-1, N}\right]$, the intersection product is given by

$$
\left[\kappa_{r, N}\right]=\theta_{N}^{N-r} \quad(0 \leqq r \leqq N) .
$$

For $[\eta] \in \mathscr{N}_{i}(B O(n))$, the formula $J_{*}^{m}[\eta]=\sum_{r=0}^{N} \alpha_{N-r, m}\left[\kappa_{r, N}\right]$ holds for a unique collection of $\alpha_{N-r, m}$ in $\mathscr{N}_{N-r}(N=i+n+m-1)$. By (4.1) and (4.2), $\alpha_{N-r, m}=\alpha_{N-r-1, m-1}$, hence we can let $\alpha_{r}=\alpha_{N-r, m} \in \mathscr{N}_{r}$. Using (4.3), we obtain the formula

$$
J \iota_{*}^{m}[\eta]=\sum_{r=0}^{N} \alpha_{r} \theta_{N}^{r}
$$

Let $m \rightarrow \infty$. Let $[\eta]_{\infty}=\operatorname{inj} \lim \iota_{*}^{m}[\eta]$ and $\theta=\operatorname{proj} \lim \theta_{N}$. Then

defines Boardman's $J^{\prime}$.

$$
J^{\prime}[\eta]_{\infty}=\sum_{r=0}^{\infty} \alpha_{r} \theta^{r}
$$

1.5'. THEOREM. $\tilde{J}=J^{\prime} \iota_{*} \nu$.

Proof. We must establish that for each $\left(M^{n}, T\right)$ and $m \geqq 1$,

$$
J \iota_{*}^{m} \nu[M, T]=\sum_{r=0}^{m-1}\left(\varepsilon \Gamma^{r}[M, T]\right)\left[\kappa_{m-r-1, n+m-1}\right] .
$$


For $m=1,(4.4)$ is true by [3, Theorem 24.2]. Note that the pair $\left(M^{n+1}, T_{3}\right)$ constructed in the proof of [3, Theorem 24.2] is simply the $\left(M^{\prime}, T^{\prime}\right)$ of $\S 2$. Thus, iterating $(m-1)$ times the proof of [3, Theorem 24.2], we establish (4.4) for $m$. Hence Theorem $1.5^{\prime}$ is established.

There is another, more geometric connection between $\tilde{J}[M, T]$ and $v[M, T]$. Let $v$ be the normal bundle over the fixed-point set $F$ of $\left(M^{n}, T\right)$. Let $R P_{r}(v)$ be the total space of the associated projective bundle of $v \oplus o^{r}$. The element $\left[R P_{r}(\nu)\right] \in \mathscr{N}_{r+n-1}$ is well determined by the element $[M, T] \in$ $\mathscr{I}_{n}$. Let

Let

$$
K[M, T]=\sum_{r=0}^{\infty}\left[R P_{r}(\nu)\right] \theta^{r+n-1} \in \mathscr{N}[[\theta]]
$$

$$
P=\sum_{r=0}^{\infty}\left[R P^{2 r}\right] \theta^{2 r} \in \mathscr{N}[[\theta]] .
$$

Proposition. $\tilde{J}=P^{-1} \cdot K$.

Proof. It is an immediate consequence of [4, Theorem 4.1].

\section{REFERENCES}

1. J. M. Boardman, On manifolds with involution, Bull. Amer. Math. Soc. 73 (1967), 136-138. MR 34 \#5093.

2. P. E. Conner, Lectures on Boardman's theory, Mimeographed, University of Virginia, Charlottesville, Va., 1966.

3. P. E. Conner and E. E. Floyd, Differentiable periodic maps, Ergebnisse der Mathematik und ihrer Grenzgebiete, N.F., Band 33, Academic Press, New York; SpringerVerlag, Berlin, 1964. MR 31 \#750.

4. - Fibering within a cobordism class, Michigan Math. J. 12 (1965), 33-47. MR 31 \#4038.

Department of Mathematics, University of Maryland, College Park, MaryLAND 20742 